Cuadernos de Lingüística Hispánica N. ${ }^{\circ} 22$ ISSN 0121-053X

Julio-Diciembre 2013; pp. 75-89

\title{
Normalización y transgresión a través de la mirada del mito*
}

\author{
LILIANA PAOLA GARCÍA MONROY**
}

paog7335@hotmail.com

Recepción: 14 de abril del 2013

Aprobación: 8 de julio de 2013

* Artículo que contiene resultados preliminares del proyecto de investigación «Normalización y transgresión a través de la mirada del mito", del grupo de investigación Senderos del Lenguaje, Universidad Pedagógica y Tecnológica de Colombia.

** Licenciada en Idiomas Modernos de la Universidad Pedagógica y Tecnológica de Colombia. Estudiante de Maestría en Literatura de la Universidad Pedagógica y Tecnológica de Colombia. Investigadora del Lenguaje en relación con el sujeto. 


\title{
Resumen
}

El presente artículo se constituye como la hoja de ruta del proyecto de investigación titulado «Normalización y transgresión a través de la mirada del mito». Se pretende mostrar una red de conexiones entre las pretensiones y especificidades de la investigación y el estado del arte y demás teorías que se convierten en la base para el desarrollo del proyecto. De esta manera, y a través de las relaciones entre lo anteriormente mencionado, se espera determinar las manifestaciones de normalización y transgresión en estudiantes de secundaria, relacionadas con su voz y su cuerpo, a través del diálogo con el mito. Dichas nociones de normalización y transgresión surgen de lo ya establecido por Michael Foucault y se conectan, en este caso, con las particularidades del contexto educativo en el cual esta investigación toma lugar.

Palabras clave: normalización, transgresión, mito, sujeto, voz, cuerpo.

\section{Normalization and transgression through the view of Myth}

\begin{abstract}
This article established the guidelines for the research project entitled «Normalization and transgression through the view of Myth». The aim is to establish connections between the intentions and specificities of the research, the state of the art and other theories on which the development of this project is based. The manifestations of normalization and transgression between secondary school students will be determined through the relations between these aspects. These manifestations are related to students' voice and body, in the process of engaging in dialogue with myth. Such notions of normalization and transgression come from Michael Foucault, and are connected in this case with the particularities of the educational context in which this research takes place.
\end{abstract}

Key words: Normalization, transgression, myth, subject, voice, body. 


\section{Normalisation et transgression à travers du regard tu mythe}

\section{Résumé}

Cet article se constitue comme la feuille de route du projet de recherche appelé «Normalisation et transgression à travers le regard du mythe ». On prétend créer un réseau de connexions entre les prétentions et spécificités de la recherche et l'état de l'art et d'autres théories qui deviennent la vase pour le développement du projet. De cette manière et à travers les rapports entre ce qui a été mentionné ci-dessus, on espère déterminer les manifestations de normalisation et de transgressions chez des élèves de l'école secondaire, reliées avec leurs voix et leurs corps, à travers le dialogue avec le mythe. Ces notions de normalisation et de transgression surgissent de ce qui a déjà été établi par Michel Foucault et se connectent, dans ce cas, avec les particularités du contexte éducatif dans lequel cette recherche a lieu.

Mots clés: Normalisation, transgression, mythe, sujet, voix, corps.

\section{Normalização, transgressão a través do olhar do mito}

\section{Resumo}

0 presente artigo se constituiu como a carta de navegação do projeto de pesquisa «Normalização e transgressão através da mirada do mito». Pretende-se criar uma rede de conexões entre as pretensões e especificidades da pesquisa e o estado da arte e as outras teorias se convertem na base para o desenvolvimento do projeto. Desta forma, e a traves das relações entre 0 falado anteriormente, espera-se determinar as manifestações de normalização e transgressão em alunos de ensino médio, relacionadas com a sua voz e seu corpo, em dialogo com o mito. Ditas noções de normalização e transgressão surgem do estabelecido por Michael Foucault e conectam-se, neste fato, com as particularidades do contexto educacional no qual a pesquisa assume o lugar.

Palabras chave: Normalização, transgressão, mito, sujeito, voz, corpo. 
El cuerpo está como separado de la experiencia, anestesiado a efectos de la convivencia de heterogéneos y, por tanto, sordo a la exigencia de creación de sentido para problemas singulares.

Suely Rolnik.

\section{Introducción}

El objetivo de este artículo está dirigido a mostrar una red de conexiones entre los distintos componentes que hacen parte del proyecto de investigación intitulado «Normalización y transgresión a través de la mirada del mito». Con el fin de generar dicha red, se hace necesario retomar el tema y la problemática de esta propuesta y los demás elementos básicos que la fundamentan, como lo son los antecedentes y la metodología adoptada dentro del proceso de investigación.

Pues bien, el título de este trabajo investigativo deja traslucir algunas palabras clave de este proceso. Dichas palabras se unen al tema central, siendo este la búsqueda del cuerpo y la voz del sujeto, a través del diálogo con el mito, bajo el panorama de la normalización y la transgresión. Con esto quiero decir que el mito, siendo una narración arquetípica, se convierte en el medio a través del cual el sujeto puede llegar a reconocer su cuerpo y su voz y, reconocer así, la posible normalización y transgresión de su cuerpo. La normalización y la transgresión se entenderán en relación con la construcción del sujeto al ser parte de una cultura.

De acuerdo con lo anterior, este proyecto pretende determinar, a través del diálogo con el mito, las condiciones del cuerpo y la voz del sujeto bajo el panorama mencionado anteriormente. De este cuestionamiento es importante resaltar que la investigación tomará lugar dentro de un contexto educativo, específicamente con un grupo de estudiantes de Grado Décimo del Colegio Andino de Tunja, institución educativa de carácter privado. Cabe especificar que, además de tomar al estudiante como sujeto de estudio, también entra la voz de la investigadora en cuanto narradora de las experiencias del otro y las propias.

De esta manera, al plantear que en este proyecto se trabajará con la idea de sujeto y que, además, este sujeto estará enmarcado en un contexto educativo formal, es conveniente entender que esa idea de sujeto no deja de lado su dimensión singular y social. Dicho de otro modo, no se pretende enmarcar al sujeto dentro de la visión estrictamente académica, sino que se espera dar cabida a cada una de sus dimensiones, entendiendo que entra en juego, también, su relación con el otro y consigo mismo. 
Por lo tanto, la concepción de sujeto que aquí se constituye es aquella que, basada en las ideas de código moral del comportamiento y formas de subjetivación de Michel Foucault, lo determina como una construcción de sí mismo, entendiendo la presencia de su cuerpo atravesado por el deseo y la norma. Así mismo, es importante para el proyecto articular la idea de la voz al sujeto, en cuanto a cómo ha sido normalizada y qué rasgos de transgresión puede tener frente al efecto de la norma. Todas estas ideas, tanto las propias como las del autor mencionado, se retomarán más adelante al hablar de las teorías base de esta propuesta.

Creo conveniente desde ahora explicitar la relevancia del mito dentro de la investigación, y así entender por qué esta narración literaria y no otra. El mito está constituido por estructuras opuestas que hacen parte de los imaginarios de los grupos sociales. De esta manera, el mito surge como una oportunidad de pensar sobre el origen de los comportamientos que no corresponden a lo que se espera socialmente del sujeto, así como de aquellos que sí encajan. Aquellos comportamientos que hacen al cuerpo diferente en un mundo de cuerpos, así como los que hacen ver al cuerpo como ser normalizado.

Por lo anterior, se espera mediante este proyecto reconocer el cuerpo y la voz del sujeto, a través de su diálogo con el mito. De esta manera, se podrá identificar los rasgos de normalización y trasgresión, producto de la inmersión en la cultura, con el fin de generar futuros espacios de reflexión sobre la necesidad de dar lugar a las prácticas de sí, comprendiendo la relación entre la normalización y la subjetividad en la construcción del sujeto. Dichos espacios se hacen necesarios en ámbitos educativos, ya que son instituciones que, generalmente, producen normalización al buscar docilidad y productividad. Ahora bien, no se trata de mejorar o no la calidad de vida del sujeto estudiado, sino de abrir espacios para que tanto estudiantes como docentes reflexionen en torno de su experiencia, usando distintas estrategias de interpretación.

Producto de la reflexión anteriormente mencionada, no se puede negar mi presencia como sujeto-investigador, ya que también se trata de un giro de la mirada hacia mí misma, entendiendo qué me ocurre en el proceso, qué sucede con mi propio diálogo con el mito y qué puedo establecer de mi relación con los sujetos investigados y con la normalización y transgresión de mi propio cuerpo y de mi voz.

Hasta ahora he mencionado las pautas mínimas que constituyen este proyecto. Sin embargo, es necesario hablar de los trabajos previos en relación con los temas que hacen parte de este proceso investigativo, es decir, en relación con el mito, el sujeto y la idea de normalización y transgresión. Es por esto que, a continuación, se mencionarán dichos trabajos, teniendo en cuenta la relación que puede existir con el presente proyecto. 
Iniciaré hablando del mito y los estudios que alrededor de este han tomado lugar. Dentro de la búsqueda de dichos trabajos, ha sido interesante encontrar tesis de estudiantes que también han visto en el mito la posibilidad de una dualidad entre elementos que parecerían incompatibles. Ejemplo de lo anterior es el trabajo para la obtención de grado de doctor de Francisco Javier Sánchez (2004), intitulado Terror y placer: bacia una (re) construcción cultural del mito y su proyección sobre lo femenino en la literatura escrita en lengua inglesa. Uno de los objetivos del trabajo consiste en establecer un paralelo entre el mito del vampiro y su concepción cultural, dejando ver la dualidad entre el terror y el placer. Esto hace ver que la mirada hacia el mito está relacionada con la cultura y, por lo tanto, con los sujetos que de ella hacen parte. Además, permite el trabajo con una dualidad, lo que también hace parte del presente trabajo de investigación, en este caso entre normalización y transgresión.

Otro de los trabajos relacionados con el mito es el de Natalia González, intitulado Adán y Eva, Fausto y Dorian Gray: tres mitos de transgresión. En esta tesis, se evidencia la manera en que los mitos mencionados poseen elementos transgresores en ideas como el bien y el mal, el castigo y la salvación, entre otras. Eso es una muestra más de que el mito es una oportunidad para trabajar aquello que, como la moneda, se constituye por dos caras. En este caso, además, aparece la palabra transgresión, entendida como la idea de elementos «corruptores» dentro de ciertos fundamentos sociales previamente establecidos.

Existen otros tipos de trabajos en cuanto a reconstrucción de mitos a partir de los ya existentes. Este es el caso de Clarissa Pinkola en su libro Mujeres que corren con los lobos (1995). Desde este punto de vista, la reconstrucción del mito está relacionada con la experiencia del sujeto, hasta el punto de develar el mito que cada quien ha construido en su vida, al ser parte de grupos sociales y culturales variados.

Continuando con la mirada hacia los trabajos ya existentes, relacionados con los mitos, se encuentran los talleres de la docente Ellie Epp, en cuanto al mito de Orfeo y Eurídice y su relación con el sujeto. Estos talleres relacionan las estructuras del mito, sus contradicciones, sus opuestos, con las del sujeto, comprendiendo que dichas estructuras se arman y se desarman con el tiempo. El trabajo de esta docente guió, en gran parte, la iniciativa de este proyecto de investigación, puesto que me permitió repensar la normalización del cuerpo en relación con la cultura y las maneras que tiene el sujeto de transgredir esa normalización.

En cuanto al sujeto y cuerpo, otro de los trabajos que se constituye como antecedente de este proceso investigativo es Lenguaje y Paz .

\footnotetext{
1 Investigadora principal: Magíster Juliana Borrero. Estudiantes auxiliares de investigación: Mónica Ardila, Melissa Mateus, Andrea Vargas, Gina Brijaldo, Adela Ávila, Carolina Peña, Gloria González, Deicy Mariana Pérez, Libardo Cortés, Lizzbeth Sánchez y Rocío Gavilán.
} 
El Proyecto Lenguaje y Paz, iniciado en Tunja en 2004, es un espacio para la exploración del cuerpo en el lenguaje, y las implicaciones de esta práctica en el cuerpo social. Es un proyecto que se inspira en la literatura -en las experiencias de descubrimiento que ha dejado la lectura de la literatura en nosotros-; y, a través de la exploración de la escritura para dar respuesta a nuestras preguntas por el ser, termina por plantear la literatura como práctica del cuerpo. Esta práctica regenera no sólo para la disciplina de la literatura, sino el trabajo de las humanidades y la investigación humanística en general (Borrero, 2010, p. 15).

A través de este proyecto algunos de los estudiantes auxiliares produjeron sus trabajos investigativos de pregrado. Es uno de ellos, Cuerpo en exilio: baciendo convivencia en la lengua de los invisibles, de las docentes Yormary Edith Torres Núñez, Heidy Jazmín Velásquez Muñoz y Liliana Inés Ruiz Rincón (2007), donde se toma la noción de sujeto en relación con la norma, dando a entender que solo un sujeto ético preocupado de sí mismo y de los otros puede entender la importancia de reaccionar ante las formas de poder que subyugan $\mathrm{y}$ anulan al sujeto.

Finalmente, hablando de la idea de normalización y transgresión, se ha constituido en la Universidad de Chile un grupo de investigación llamado Disciplinamiento/ normalización: núcleo de la sociología del cuerpo. Este grupo se ha concentrado en un «debate sobre todos los lugares, espacios, dispositivos y tecnologías históricas y contemporáneas, a través de las cuales los cuerpos son moldeados, producidos y posibilitados de participar de la realidad cotidiana» (Universidad de Chile, 2011). Otro de los temas de interés de este grupo, muy ligado al anterior, es el relacionado con las instancias, no solo de dominación, sino también de resistencia en las que los cuerpos se desenvuelven. Esto último se constituye como una de las bases de mi investigación, al pensar la normalización y la transgresión (formas de resistencia) del sujeto frente a su cuerpo y su voz.

\section{Metodología}

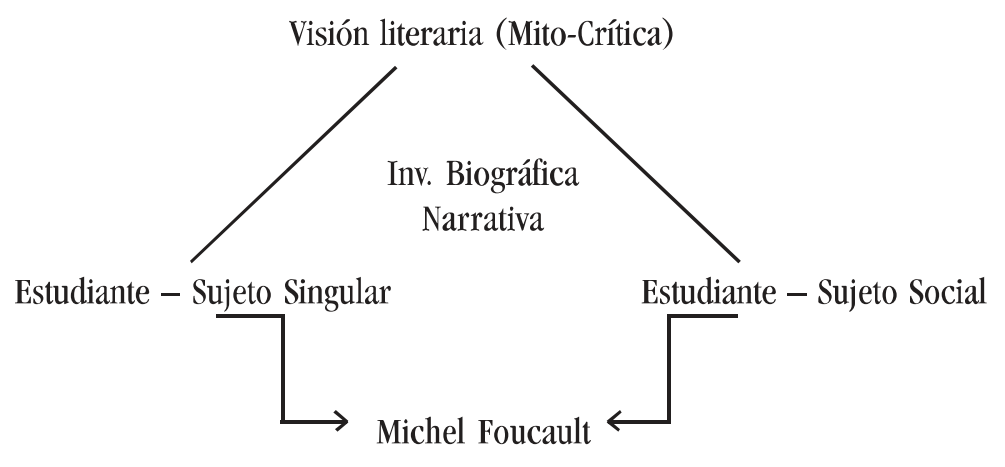


Con el fin de dar a conocer el diseño metodológico de esta investigación, trataré de esbozar el planteamiento del mismo con base en el anterior gráfico. Es de aclarar que no existe para este proceso un diseño predispuesto en el que ya todo esté dicho. En otras palabras, el proceso metodológico hace parte de la construcción de este proyecto, que parte de la Investigación Biográfica Narrativa (IBN).

Este tipo de investigación ha sido utilizada frecuentemente en educación, y pretende «ampliar el conocimiento sobre lo que realmente sucede en el mundo escolar, a través del punto de vista de los implicados [...] en una mirada personal e íntima de su proceso educativo, recuperando su propia voz al hacerla pública» (García, Lubián, \& Moreno, (s.f.). Algunos de los instrumentos de recolección de datos que permiten el análisis de las experiencias propias del sujeto son las notas de campo, diarios, transcripción de entrevistas, relatos, cartas, escritos autobiográficos y biográficos, carpetas de aprendizaje o portafolios, otros documentos como planeaciones de clase o boletines, escritos tales como normas, o reglamentos, y fotografías, entre otros.

Ahora bien, al ver que la IBN permite analizar experiencias es necesario determinar a qué tipo de «experiencia» nos estamos refiriendo. Es por esto que en la metodología del proyecto, este concepto tiene que ver con lo propuesto por Michel Foucault (2005) en cuanto a su genealogía del deseo, el cual se basa en la experiencia como la «correlación en una cultura, entre campos de saber, tipos de normatividad y formas de subjetividad» (p. 8), elementos que, más adelante, podrían hacer parte de una triangulación de perspectivas. Dicho de esta manera, la experiencia (desde Foucault) y su narración (desde la IBN) son la base del diseño metodológico, al entender que, siendo el sujeto y su relación con el lenguaje el eje del proyecto, son sus experiencias y prácticas el objeto de análisis.

Sin embargo, al ser este un estudio de tipo literario se hace totalmente necesario recalcar la visión que de este campo se tiene. Al ver la gráfica anterior, nos damos cuenta que en la punta se encuentra la mito-crítica, propuesta de crítica literaria de Gilbert Durand, (1993), la cual, unida con el mito-análisis, supone que es posible encontrar en el mito un espacio para el imaginario cargado de un potencial simbólico propio de una sociedad. Además, Durand habla de la posibilidad de encontrar unos mitemas dentro del mito, los cuales poseen una capacidad simbólica que tiene que ver con dualidades humanas. De esta manera, se hace posible determinar esos campos simbólicos en el mito y relacionarlos con el sujeto, en este caso, el estudiante y el docente.

Este proyecto se realizará con estudiantes de Grado Décimo del colegio ya mencionado. Sin embargo, es importante aclarar que no se trabajará con todo el curso, sino con solo 
una muestra de 5 estudiantes, elegidos al azar. Además, mi práctica docente también entra a ser parte del proceso de investigación, no solo en la dimensión laboral, sino también siendo sujeto singular y social.

A continuación, se presenta la manera en que las teorías centrales del proyecto, es decir, la normalización y la transgresión, entran en diálogo con la idea de cuerpo, voz y mito.

\section{Normalización y transgresión a la luz de las teorías}

Considero pertinente iniciar aclarando brevemente las concepciones relevantes que hacen parte del proyecto, con el fin de facilitar el recorrido de las líneas que vienen a continuación.

Las concepciones de normalización y transgresión, nociones previamente mencionadas, tiene origen en las ideas de Michel Foucault (2005) cuando habla de «código de comportamiento (normas y encauzamiento de conductas) y las formas de subjetivación o prácticas de sí -relación consigo mismo-» (p. 30). Es así como la normalización se relaciona con términos como la disciplina, el control, las normas y los medios de encauzamiento de conductas, y las formas de subjetivación tienen que ver con lo que se queda por fuera de ese control de conductas, con las desviaciones que hacen al sujeto singular y que lo llevan a reconocer-se y a reconocer al otro.

El cuerpo se entiende, a partir de la normalización y transgresión, como aquel que ha sido parte de la sistematización de la norma hasta el punto de sistematizarse a sí mismo, perdiendo contacto con su subjetividad. Ubico esta concepción en el contexto específico de la escuela, donde, tal vez, es uno de los espacios normalizadores por excelencia. En relación con lo anterior, Suely Rolnik (2011) afirma que el cuerpo está alejado de toda experiencia singular, ya que siempre ha estado acostumbrado a alimentarse de una cultura «hegemónica», la cual le evita construir sentidos propios:

El cuerpo está como separado de la experiencia, anestesiado a efectos de la convivencia de heterogéneos y, por lo tanto, sordo a la exigencia de creación de sentido para los problemas singulares que se esbozan en esta exposición. La tendencia que se mantiene hegemónica desde entonces es la de consumir cultura europea, una cartografía de sentido que, además de haberse producido en una experiencia de no mixtura, está desencarnada de la experiencia sensible por haberse forjado bajo la égida del racionalismo (p. 33).

Es por esto que el sujeto necesita reconocer su cuerpo para poder reconocer su singularidad. Además, necesita entender que su paso por la cultura ha demarcado un 
sinnúmero de normas que determinan sus experiencias o vivencias. Lo anterior no quiere decir que no haya rasgos de transgresión en el cuerpo, sino que es uno de los intereses de este proyecto poder determinarlos.

De modo similar se concibe la voz. No es la voz como producción fónica, sino la voz como postura ética y política del sujeto. La que le permite dejar de repetir discursos vacíos por no ser propios y concebir-se en relación consigo mismo y con el otro. Quisiera retomar a Carole Gilligan (2003), quien a través de su interés por la diferencia entre amor y democracia se pregunta por la voz perdida que debe ser recuperada:

Me pregunto por la relación entre amor y democracia, la unión íntima entre vida pública y privada. Tanto el amor como la democracia dependen de que la voz sea voz y también de la resonancia que hace posible hablar y ser escuchado. Sin voz, no hay relaciones entre las personas; sin resonancia, la voz se convierte en silencio (p. 30).

Es la voz que, al entrar en diálogo con el cuerpo y su subjetividad, se convierte en propia.

Ya para finalizar con estas breves miradas hacia términos que son la base del proceso, quisiera hablar brevemente de la manera en que el mito se convierte en un eje del objetivo principal de esta investigación. El mito es una construcción arquetípica que permite descifrar dualidades humanas, generalmente disociadas por la normalización y la transgresión. Según el antropólogo Claude Levi-Strauss (1995), «todo mito cumple con tres atributos: trata de una pregunta existencial, está constituido por contrarios irreconciliables y proporciona la reconciliación de esos polos para poner fin a la angustia» (p. 235). Entender el sujeto como una construcción entre la normalización y la transgresión, implica reconocer esas dualidades que hacen parte de él y lograr, a través del mito, un equilibrio entre tales contradicciones. Equilibrio encaminado al reconocimiento del cuerpo en reconciliación con otros cuerpos.

Y es precisamente con estas dos nociones, normalización y transgresión, con las que quisiera iniciar el planteamiento de las teorías que soportan este proyecto. Para tal fin, debo iniciar hablando, primero, del código moral de los comportamientos y, luego, de las formas de subjetivación o prácticas de sí según Michel Foucault.

Para Foucault (2005), la normalización del cuerpo inicia con las formas de disciplina que se empleaban en los procesos de docilidad con los soldados. De esta manera, se consideraba que un cuerpo era dócil en la medida en que podía ser sometido, utilizado, transformado y perfeccionado. Foucault (1976) explica la manera en que esos sistemas de disciplina fueron retomados en las instituciones educativas, con el fin de «aumentar las 
fuerzas del cuerpo (en términos económicos de utilidad) y disminuir esas mismas fuerzas (en términos políticos de obediencia)» (p. 83). Es por esto que se considera que, en ambientes de disciplina y normalización como las instituciones educativas, se busca generar una alta productividad por parte del sujeto o estudiante bajo el régimen de la obediencia, dejando de lado su subjetividad atravesada por el deseo.

Foucault habla además, de las maneras en que las distribuciones de la disciplina generan cierto tipo de normalización. En el caso particular de los colegios, se distribuye a los individuos en un espacio de clausura, en el que es más sencillo controlar las actividades de aquellos que hacen parte del sistema. A toda esta distribución, en la que se especifican, además, conventos, cuarteles, entre otros lugares, Foucault la llama $\mathrm{El}$ arte de las distribuciones. Es así como el espacio físico en el que se lleva a cabo el proceso de normalización entra en relación con los sujetos que allí habitan, esos ambientes cerrados facilitan el control.

A la hora de ejercer dicho control sobre las actividades propias de las instituciones educativas entra en juego la noción de tiempo. Según Foucault (1976), en la escuela se «define una especie de esquema anatomo-cronológico del comportamiento [...] El tiempo penetra el cuerpo y con él todos los controles minuciosos del poder» (p. 92). Esto quiere decir que cuando el sujeto se ve obligado a establecer ritmos de trabajo, a ocupar obligaciones determinadas y a regular ciertos ciclos de repetición, su cuerpo entra en juego con el tiempo, haciendo que este último llegue a controlarlo en función de la productividad.

Lo anterior es una explicación de cómo en los colegios se utiliza la fragmentación del tiempo en función del control de las actividades del estudiante. De esta manera, el sujeto se acostumbra a ciertos ritmos de producción y siente angustia si no cumple con esos regímenes de trabajo. Ahora bien, la repetición de esos ciclos de producción hace que el estudiante duerma su cuerpo frente al panorama de las actividades por desarrollar. Es decir, al ser un proceso tan sistemático, no hay cabida para la subjetividad dentro del proceso de control.

Ahora bien, al ser la disciplina la encauzadora de comportamientos o conductas, existen, según Foucault, medios del buen encauzamiento, los cuales son utilizados en instituciones por naturaleza normalizadoras, como lo es la escuela. Uno de esos medios es la vigilancia jerárquica, a través de la cual, y gracias al arte de la distribución, distintos agentes ejercen control sobre las actividades de quienes hacen parte del sistema. Esta vigilancia hace parte de todo el proceso de producción, en el que cada agente cumple su función. Foucault habla de cómo el maestro, para facilitar su tarea, asignaba a sus estudiantes 
distintos roles que le ayudaran a mantener el control dentro del aula de clase. Solo uno de esos roles asignados tenía que ver con la pedagogía, los demás ejercían control disciplinario. Aquel que ejercía la función pedagógica era el «repetidor», haciendo que sus compañeros leyeran, como su nombre lo indica, de forma repetitiva.

Es aquí donde se puede notar el distanciamiento del sujeto de su cuerpo y su voz, ya que al ser obligado a regular ciclos de repetición, no hay espacio alguno para girar la mirada hacia sí mismo, y tener la oportunidad de reconocerse y, por ende, reconocer al otro. Poco a poco se determina a través de las teorías de Foucault la manera en que la normalización, a través del código moral de los comportamientos, lleva al sujeto a alejarse de las prácticas de sí, en función de su auto-reconocimiento y transformación.

Continuando con los medios del buen encauzamiento, otra de las formas de disciplina de las conductas es el uso de la sanción normalizadora, según la cual existe una micropenalidad del tiempo y de la actividad, como ya se había mencionado anteriormente, pero también existe una micropenalidad de la palabra y del cuerpo. Esta forma de sanción castiga «la inobservancia, todo lo que no se ajusta a la regla, todo lo que se aleja de ella, las desviaciones». En el caso de la voz, se castiga «la insolencia y la charla», en el caso del cuerpo, «las actitudes incorrectas, gestos impertinentes y la suciedad». Esto vuelve a plantear la idea de cómo el cuerpo y la voz propia son suprimidos de los procesos de normalización en la escuela, al considerarse «desviaciones» y al ser, como tal, formas de subjetivación 0 prácticas de sí, en términos de Foucault.

El tercero y último modo del buen encauzamiento corresponde al examen. Este modo ha sido visto, desde una mirada normalizadora, como la manera de «calificar, clasificar y castigar» (Foucault M. , 1976, p. 113). Es así como el examen se convierte en otra forma de estandarizar. Al implicar castigo, continúa con la normalización tanto del cuerpo como de la voz, lo que hace que el examen se convierta para el sujeto en un discurso vacío.

Por otro lado, y refiriéndome ya a las formas de subjetivación o prácticas de sí, Foucault, en el prólogo a la Historia de la sexualidad: El uso de los placeres, hace referencia a cómo en el paganismo, la moral circundante era la de la ascesis o artes de la existencia que según él son «prácticas reflexivas y voluntarias por las que los hombres no solo se fijan reglas de conducta, sino que intentan transformarse a sí mismo, modificarse en su ser singular» (Foucault M. , 2005, p. 14). De esta manera, se recuerda en la historia al sujeto en sus prácticas de sí. Sin embargo, con la llegada del cristianismo, esas prácticas fueron aplacadas para dar paso a la moral del código de los comportamientos.

\section{6}


Lo anterior no significa que el sujeto haya suprimido por completo esas formas de subjetivación. Lo que sí es cierto es que esas formas se consideran ahora como líneas de escape o de fuga a la normalización circundante. La manera en la que el sujeto asume la norma se ha llamado por Foucault (2005) como los modos de sujeción, siendo la forma en la que el individuo establece su relación con la norma y «se reconoce como vinculado con la obligación de ponerla en obra» (p. 27).

Po lo tanto, en el campo de las prácticas de sí, se concibe al sujeto en posición frente a la norma, siendo él quien asume de distintas maneras tal posición. Ahora bien, son esas posturas diferentes a las normalizadas las que son ahora consideradas como desviaciones, al ser parte de la subjetividad.

Pues bien, para no ir más allá, las formas de subjetivación se relacionan con las maneras en que los sujetos se relacionan consigo mismos, «los procedimientos y las técnicas mediante las cuales se las elabora, sobre los ejercicios mediante los cuales uno se da a sí mismo como objeto de conocimiento y sobre las prácticas que permiten transformar su propio modo de ser» (Foucault M. , 2005, p. 31).

Dicho en otras palabras, se trata de girar la mirada hacia el sujeto mismo, hacia sus prácticas, hacia su cuerpo y su voz. Es poder determinar cuáles son esas «desviaciones» que hacen al sujeto diferente y que lo llevan a ser su propio objeto de conocimiento.

Líneas más arriba, en el campo de la normalización, se hablaba de los medios del buen encauzamiento. Pues bien, existen en el campo de las prácticas de sí o de la transgresión, los medios de subjetivación. Estos medios son el de la sustancia ética, los tipos de sujeción y las formas de elaboración de sí. La sustancia ética tiene que ver con los actos deseados y asociados a un placer intenso. Los tipos de sujeción se refieren al principio a través del cual se regula la sustancia ética, no en términos de lo permitido y lo prohibido, sino más bien por la prescripción de las modalidades de un uso en función de variables diversas (necesidades, momento, situación). Finalmente, las formas de elaboración de sí se refieren a la manera en que el sujeto encuentra el equilibrio entre lo social y lo singular, sin llegar a desconocer ninguna de las partes. Esto, según Foucault, se constituye como la única manera de encontrarse a sí mismo y, por consiguiente, al otro.

Puesto que ya se ha aclarado en gran medida la forma en que las teorías entran en diálogo entre sí, es hora de retomar la idea de mito dentro de todo el panorama de normalización y transgresión del sujeto. Es por esto que, a continuación, se hablará del mito y su relación con toda la problemática. 


\section{El mito: búsqueda del cuerpo y la voz del sujeto.}

La concepción de mito que atañe a esta investigación, está relacionada con su atemporalidad, es decir, el mito es una narración que siempre está vigente, aun cuando su origen sea impreciso. Según Mircea Eliade (1991), «el mito cuenta cómo, gracias a las hazañas de los seres sobrenaturales, una realidad ha venido a la existencia, sea esta la realidad total, el cosmos, o solamente un fragmento: una isla, una especie vegetal, un "comportamiento humano, una institución» (p. 3). Con base en lo anterior, el mito es una narración que cobra vida a la luz de los comportamientos humanos, en relación con todo lo que rodea al sujeto.

De esta manera, el mito se convierte en una herramienta de diálogo, a través de la cual el sujeto puede llegar a identificar ciertas estructuras que lo establecen como ser individual y social. Esto es aún más evidente, al entender el mito como una narración arquetípica, de acuerdo con la cual, y recordando la teoría del inconsciente colectivo de Carl Jung, el sujeto busca crear representaciones y confirmarlas a través de lo que está a su alrededor, incluyendo lo que encuentra en narraciones como el mito. De acuerdo con Rosario Scrimieri (2008), para Jung «tienen que haber mitos típicos, verdaderos instrumentos que sirvan a los pueblos para elaborar sus complejos psicológicos y sus conflictos» (p. 95); de ahí su relación con los sujetos a los que alude.

Los mitos tocan temas que forman parte de la herencia colectiva de la humanidad. Son narraciones simbólicas que pueden llegar a no ser comprendidas. A través de los símbolos que hacen parte del mito, este puede reflejar algo importante para el sujeto, quien capta y ve una verdad a través de ellos.

Ahora bien, hablar de sujeto en esta investigación no limita la visión que de él se tiene socialmente. Dicho en otras palabras, el sujeto es, porque está en relación con otro en medio de experiencias socio-culturales que lo determinan por su rol en el grupo al cual pertenece. Es por esto que el mito no desconoce la importancia de la sociedad para el sujeto, y mucho menos todas las manifestaciones culturales que lo rodean. Para Joseph Campbell (1959), el mito cobra importancia desde el cuerpo y la mente humana hasta todo aquello que hace parte de sus experiencias, en relación con instituciones sociales:

En todo el mundo habitado, en todos los tiempos y en todas las circunstancias, han florecido los mitos del hombre; han sido la inspiración viva de todo lo que haya podido surgir de las actividades del cuerpo y de la mente humana. No sería exagerado decir que el mito es la entrada secreta, por la cual las inagotables energías del cosmos se vierten sobre las manifestaciones culturales humanas. Las religiones, las filosofías, 
las artes, las formas sociales del hombre primitivo e histórico, los primeros descubrimientos, científicos y tecnológicos, las propias visiones que atormentan el sueño, emanan del fundamental anillo mágico del mito (p. 10).

Por lo anterior, es innegable la relación entre el mito y el sujeto, no solo en su subjetividad, sino también como ser socio-cultural. Esta narración mítica ha acompañado al hombre desde sus inicios y, por lo tanto, no se debe desconocer la relación que entre sujeto y literatura, en este caso mito, se entrelaza en estructuras, algunas de ellas como construcciones socialmente representadas.

\section{Referencias bibliográficas}

Borrero, J. (2010). La literatura como práctica del cuerpo. En: Revista La Palabra, № . 16, Universidad Pedagógica y Tecnológica de Colombia, Tunja.

Campbell, J. (1959). El héroe de las mil caras. México: Fondo de cultura económica.

Durand, G. (1993). De la mitocrítica al mitoanálisis: figuras míticas y aspectos de la obra. Barcelona: Antropos.

Eliade, M. (1991). Mito y Realidad. Barcelona: Labor.

Foucault, M. (1976). Vigilar y Castigar. México: Siglo XXI Editores.

Foucault, M. (2005). Historia de la sexualidad Tmo II. España: Siglo XXI Editores. Recuperado de http://ebookbrowse.com/michel-foucault-historia-de-la-sexualidad-tomo-iiintroduccion-1576275417-pdf-d278647387.

García, M., Lubián, P., \& Moreno, A. (s.f.). Universidad Autónoma de Madrid. Recuperado de http://www.uam.es/personal_pdi/stmaria/jmurillo/InvestigacionEE/Presentaciones/ Curso_10/IBN.pdf

Gilligan, C. (2003). The birth of pleasure. New York: Vintage books.

Levi-Strauss, C. (2004). Antropología estructural. México: Siglo Veintiuno Editores.

Pinkola Estés, C. (1995). Mujeres que corren con los lobos. Barcelona: Ediciones B, S. A.

Rolnik, S. (2001). Más allá del principio de identidad: la vacuna antropofágica. Teatro al sur. Revista Latinoamericana (20). pp. 32-39. 
Sánchez, F. (2004). Terror y placer: bacia una (re) construcción cultural del mito del vampiro y su proyección sobre lo femenino en la literatura escrita en lengua inglesa. Ciudad Real: Universidad de Castilla-La Mancha.

Scrimieri, R. (2008). Los mitos y Jung. Amaltea, pp. 87-112.

Torres, Y., Velásquez, H., \& Ruiz, L. (2007). Cuerpo en Exilio: baciendo convivencia en la lengua de los invisibles. Tesis de grado. Tunja: Universidad Pedagógica y Tecnológica de Colombia.

Universidad de Chile. (2011). Disciplinamiento/normalización: núcleo sociología del cuerpo. Recuperado de http://disciplinamientoynormalizacion.blogspot.com/ 